\title{
Experiencia Educativa con un Paciente Experto
}

\author{
Francisca Molina Escribano ${ }^{a}$, Encarna Castaño Moreno ${ }^{b}$, Javier Massó Orozco ${ }^{c}$
}

\begin{abstract}
a Médico Residente de Medicina Familiar y Comunitaria. Centro de Salud de Casas de Juan Núñez (Albacete).

${ }^{\mathrm{b}}$ Enfermera de Alatoz. Centro de Salud de Casas de Juan Núñez (Albacete).

${ }^{\mathrm{c}}$ Médico de Alatoz. Centro de Salud de Casas de Juan Núñez (Albacete).
\end{abstract}

Correspondencia: Francisca Molina Escribano. C/ Juan de Toledo $13,1^{\circ}$, 02005-Albacete. TIfno: 647930184, e-mail: paqui.molina@gmail.com

Recibido el 12 de abril de 2008.

Aceptado para su publicación el 20 de mayo de 2008.

\section{RESUMEN}

El perfil de la relación médico-paciente ha cambiado en los últimos años pasando de un modelo paternalista a un modelo en el que la autonomía del paciente ha adquirido una importancia fundamental. El paciente se está convirtiendo en un personaje activo, con unos derechos claros, como los de ser debidamente informado sobre su enfermedad o participar en las decisiones que afectan a su patología, pero también con los deberes de ser responsable de su enfermedad y de su estado de salud (1).

Presentamos una experiencia realizada en nuestra consulta acerca de un paciente que se había responsabilizado de su problema de obesidad desde que se le diagnosticó, identificando muy bien los motivos que le encaminaron a seguir nuestros indicaciones terapéuticas, las dificultades que se le fueron planteando durante el tratamiento y cómo fue resolviendo algunas dificultades

Palabras clave: Educación sanitaria.

\section{ABSTRACT}

Educational experience with the expert patient.

The doctor-patient relationship has changed considerably in recent years, from a paternalistic model to one in which the patient's autonomy has acquired a significant relevance. The patient has become an active individual, with clear rights, such as that of being well informed about his/her illness and participation in decisions that can affect their condition, but also certain obligations such as being responsible for their illness and health.

We present an experiment carried out in our clinic of a patient who had taken on responsibility for his obesity since he was diagnosed, who clearly identified the reasons for following our therapeutic indications, the difficulties he came across during the treatment and how he resolved some of them. Key words. Health education.

\section{INTRODUCCIÓN}

El perfil de la relación médico-paciente ha cambiado en los últimos años, pasando de un modelo paternalista a un modelo en el que la autonomía del paciente ha adquirido una importancia fundamental. El paciente se está convirtiendo en un personaje activo, con unos derechos claros, como los de ser debidamente informado sobre su enfermedad o participar en las decisiones que afectan a su patología, pero también con los deberes de ser responsable de su enfermedad y de su estado de salud ${ }^{1}$.

Cuando los procesos agudos eran la principal causa de enfermedad, los pacientes generalmente eran inexpertos y pasivos receptores del conocimiento médico. Ahora que las enfermedades crónicas son el principal problema de salud, el paciente debe ser un agente colaborador activo del proceso ${ }^{2}$. En España, como en todos los países desarrollados, las enfermedades crónicas son cada día más frecuentes, entre otras razones, por los cambios en los estilos de vida y por el envejecimiento de la población. Se calcula que prácticamente el $50 \%$ de esa población sufre algún tipo de enfermedad crónica para la que necesita cambios en su estilo de vida o tratamientos farmacológicos.

La educación para la salud ha sido siempre un arma terapéutica más para la prevención y el tratamiento de estas enfermedades. Hasta ahora, también siguiendo un modelo paternalista, éramos los médicos y enfermeras los encargados de impartir, exclusivamente, esta educación, partiendo de nuestros conocimientos "teóricos" sobre las enfermedades y sus tratamientos.

Desde hace unos años se está desarrollando una novedosa forma de realizar la educación para la salud que, teniendo en cuenta los principios anteriormente descritos, está basada en lo que se ha denominado "Paciente Experto". Estos programas de 
educación tratan de "utilizar" a pacientes crónicos que son capaces de entender su enfermedad, de responsabilizarse de su salud y que manejan correctamente las opciones terapéuticas marcadas por sus sanitarios. Estos pacientes, si además tienen algunas dotes comunicativas y empatía, una vez formados y asesorados, son capaces de liderar grupos de pacientes de forma eficaz, ya que trasmiten la información con el mismo lenguaje de los otros pacientes, desde la misma experiencia de enfermedad y con dificultades parecidas para la realización correcta del tratamiento y para asumir el impacto físico, emocional y social de esa enfermedad ${ }^{1}$. Los pacientes llevan la iniciativa de su formación y los profesionales sanitarios tienen un papel observador, orientador y, si es necesario, aclaran las dudas que surgen.

Las primeras experiencias con programas de pacientes expertos se llevaron a cabo en el Servicio Nacional de Salud Británico ${ }^{3}$ y en la Universidad de Stanford (EEUU), donde la investigadora Kate Lorig lleva 20 años utilizando estos programas con enfermos de artritis, artrosis, diabetes, esclerosis múltiple, obesidad, enfermedades cardíacas o pulmonares, cáncer, etc., así como con sus cuidadores o familiares.

En España el Programa Paciente Experto es una iniciativa de la Fundación Educación Salud y Sociedad, que cuenta con el apoyo de instituciones públicas y privadas a nivel nacional. Está dirigido a pacientes y sus familiares, cuidadores y profesionales. El Instituto Catalán de la Salud inició en Septiembre de 2006 programas con pacientes expertos en algunos centros de salud de Barcelona para grupos de afectados de insuficiencia cardiaca crónica.

Los objetivos de estos programas son los mismos que los de cualquier programa de educación para la salud: conseguir que el paciente tenga un mayor grado de conocimiento de su enfermedad, que sepa actuar ante un síntoma, que aprenda a interactuar positivamente sobre su entorno y que se involucre en las decisiones respecto a su enfermedad.

En los resultados ofrecidos por las pocas experiencias que hay con este sistema educativo, destacan: un aumento en la confianza del paciente para autocuidarse, mejoras en la calidad de vida y en el bienestar psicológico, incremento de la autoestima y de la "energía" y un alto grado de satisfacción con las sesiones del programa ${ }^{4,5}$.

\section{NUESTRA EXPERIENCIA}

Tras habernos informado sobres las experiencias catalanas del Programa Paciente Experto ${ }^{1}$, nos planteamos realizar una experiencia parecida en nuestra consulta.

Elegimos el tema de la obesidad por ser un problema crónico, con importante tasa de fracaso en su tratamiento y sobre el que ya habíamos ofrecido suficiente información y educación tanto en grupos, siempre dirigidos por nosotros, como a través de la educación individual en la consulta. En alguna ocasión hemos captado la idea que tienen algunos pacientes, ante nuestros consejos, de que los sanitarios "vemos muy fácil hacer lo que recomendamos porque el problema no nos afecta personalmente a nosotros".

Enseguida pensamos en el paciente que iba a ser "Experto". Se trata de un paciente que se había responsabilizado de su problema de obesidad desde que se le diagnosticó, identificando muy bien los motivos que le encaminaron a seguir nuestros indicaciones terapéuticas, las dificultades que se le fueron planteando durante el tratamiento y cómo las fue resolviendo. Este paciente ha ido consiguiendo sus objetivos de peso realizando una dieta saludable, más variada y equilibrada que la que realizaba, aumentando el ejercicio físico y realizando un cambio de actitud que le ha llevado a modificar hábitos.

El Paciente experto fue entrenado en la consulta de enfermería, donde se le explicó la idea, preparando, en dos sesiones, un esquema sobre el cual él desarrolló posteriormente su charla.

Se citó para participar en esta actividad a un grupo de 12 obesos, de los cuales acudieron 9 (8 mujeres y un hombre), con edades comprendidas entre los 40 y 60 años. Todos ellos eran obesos de larga evolución a los que se les seguía individualmente en la consulta, tanto de enfermería como de medicina, dentro del programa de control de riesgo cardiovascular.

La sesión fue participativa y se desarrolló con mucha naturalidad y con un lenguaje claro y directo para todos los participantes. Nuestro papel, como profesionales, fue de meros observadores, para después analizar la sesión con el paciente experto y con los asistentes.

Como en toda intervención en salud, es fundamental realizar una evaluación de resultados. En este caso la evaluación sólo recogió el grado de satisfacción de los participantes con la actividad, que fue muy alto. Para próximas intervenciones nos planteamos realizar encuestas de conocimientos pre y post intervención y valoraciones del impacto en salud, por ejemplo reducción de peso del grupo a los 6 meses y al año.

\section{CONCLUSIONES}

En nuestra opinión, esta nueva manera de realizar educación para la salud resulta una experiencia interesante y efectiva, en la que se podrá implicar a muchos enfermos de forma activa en su realización, sin excesivo esfuerzo por parte de los sanitarios. Además, es una actividad sencilla de realizar con la comunidad y no precisa de la utilización de demasiados recursos.

Supone una alternativa educativa que se adapta mejor al nuevo perfil del paciente, fortaleciendo su autonomía y evitando el papel de mero receptor de información, para pasar a ser un elemento clave en el cuidado de su salud.

Esta información llega de forma más directa al paciente, ya que se realiza desde un nivel similar al suyo, con una experiencia parecida y utilizando su mismo lenguaje. El paciente experto es un paciente informado, capacitado 
y comprometido con sus cuidados, contribuyendo así con sus ideas, experiencias y conocimientos al tratamiento de los demás. Todo esto puede ayudar a evitar la actitud derrotista de algunos pacientes frente al personal sanitario y a que afloren más las dudas, dificultades y preocupaciones que los pacientes tienen en relación a su enfermedad.

Pensamos que puede ser aplicada en cualquier otra patología crónica, tales como diabetes, insuficiencia cardiaca, asma, EPOC, etc., las cuales suelen presentar dificultades en su manejo en la consulta diaria.

\section{BIBLIOGRAFÍA}

1. López M. El paciente experto toma la palabra. En portada. Siete días médicos. 2007; 754:5-11.

2. Holman H, Lorig K. Patients as partners in managing chronic diseases. Partnership is a prerequisite for effective and efficient health care. BMJ. 2000; 320:526-7.

3. EPP. Expert Patients Programme. Disponible en: www.expertpatients.com.uk/public/default.aspx?loab=PublicHome.

4. Plews C. Expert Patient Programme: managing patients with long-term conditions. Br J Nurs. 2005; 14(20):1086-9.

5. Hainsworth T. A new A new model of care for people who have long-term conditions. Nurs Times. 2005; 101(3):289. 